\title{
A Methodology and Framework for the Semi-automatic Assembly of Learning Objects
}

\author{
Katrien Verbert ${ }^{1}$, David Wiley ${ }^{2}$ and Erik Duval ${ }^{1}$ \\ ${ }^{1}$ Dept. Computerwetenschappen, Katholieke Universiteit Leuven, \\ Celestijnenlaan 200A, B-3001 Leuven, Belgium \\ $\{$ Katrien.Verbert,Erik.Duval\}@cs.kuleuven.be \\ ${ }^{2}$ Instructional Design and Technology Department, \\ Brigham Young University, Provo, UT, USA \\ David.Wiley@byu.edu
}

\begin{abstract}
One of the major obstacles in developing high quality content for learning is the substantial development cost and effort. In addition, the return on investment is often low, as developed learning materials are difficult to reuse and adapt to new and different educational contexts. In this paper, we present a semi-automatic content assembly methodology to automate, at least partially, the reuse of existing learning content in high quality and effective learning sequences. In addition, we present a case study that integrates the approach into the LAMS learning design environment.
\end{abstract}

Keywords: learning object reuse, learning object metadata, learning design

\section{Introduction}

Many existing course documents merge the representation of content and the instructional approach [1]. Such hardwired pedagogy restricts the options for teaching and learning, both in terms of reusability and adaptation of learning sequences. Typically, teachers create their teaching strategies and content from scratch or reuse parts of existing course documents by ad-hoc and time-consuming copy-and-paste actions. In addition, adaptation to individual learning or teaching styles, background, experiences, interests or preferences is generally not possible, unless learning content is specifically designed for personalization purposes [2].

In this paper, we present a semi-automatic content assembly methodology for the generation of learning sequences tailored to different pedagogical approaches, based on the explicit design of these sequences by a teacher. The assembly framework employs a teacher model, an instructional model and a domain model to enable the focused retrieval and aggregation of learning resources into learning sequences. Learning resources are retrieved through the GLOBE network of educational repositories [http://globe-info.org/] and from various community driven websites, such as WikiAnswers.com, ProProfs.com and Wikipedia. The assembly framework is 
described in the next Section. We present a case study that integrates the approach into the LAMS [3] learning design environment in Section 3. Related work is discussed in Section 4, followed by conclusions and remarks on future work.

\section{Content Assembly Framework}

The content assembly framework supports the selection and assembly of existing learning resources. The framework employs the following models to enable the focused retrieval and aggregation of resources:

- The instructional model captures the semantics of the pedagogical strategy employed by a learning sequence and is based on [4]. Narrative structures within this model outline the flow of concepts of a particular learning design strategy and are used as templates when assembling learning sequences. An example is an inquiry based learning strategy that sequences activities like "answer questions", "vote on a list", "discuss responses", "read expert view", "discuss expert view" and "personal reflection".

- The domain model represents the knowledge domain of a course. It includes concepts outlined in the objectives of a course and their interrelationships.

- The teacher model defines teacher attributes to enable the personalized aggregation of learning resources [5]. The model includes attributes for representing the level of expertise of the teacher, interests and activities, teaching strategy preferences, background, and presentation styles (Fig. 1).

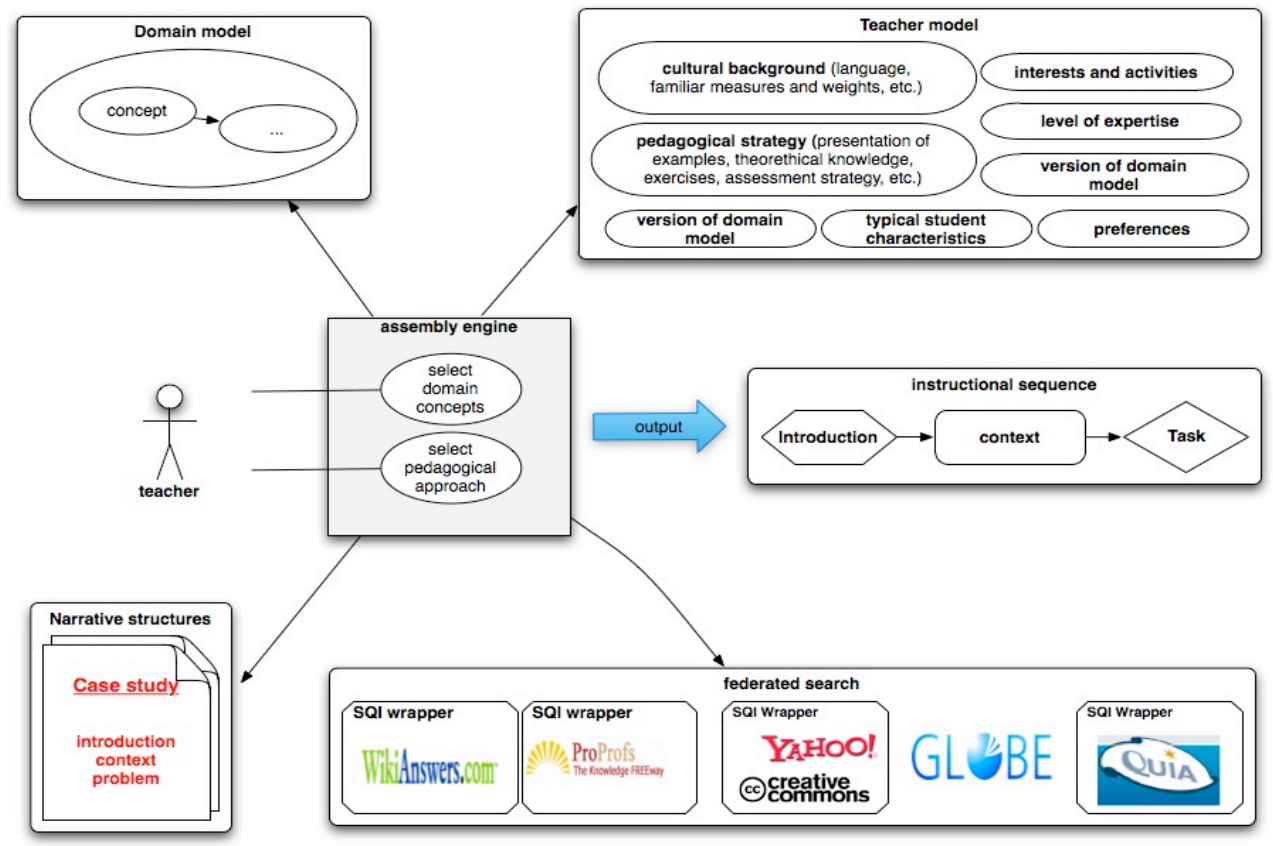

Fig. 1. Semi-automatic content assembly framework 
The assembly engine maps instructional, domain and teacher concepts to PLQL queries and federates the queries to SQI-enabled repositories. The approach is exemplified in [6]. PLQL [7] is primarily a query interchange format for repositories. SQI [8] is a query transport standard that is widely used within the technology enhanced learning community. The GLOBE alliance [http://globe-info.org/] builds on the SQI standard to enable worldwide access to learning repositories.

Moreover, to enable retrieval of relevant content resources on the Web, several SQI wrappers were built on top of community driven websites that host large amounts of content, such as WikiAnswers.com, ProProfs.com and Wikipedia. The wrappers retrieve both relevant pages and relevant fragments within the pages. The engine typically exploits the structure of pages to identify content fragments that are reusable, such as individual questions and answers of multiple choice questions or animations within HTML pages. Simple screen scraping approaches are employed to retrieve relevant parts of domain specific websites. Depending on the granularity of the narrative concept, single assets or larger compositions are retrieved, such as single questions versus entire surveys.

\section{LAMS Case Study}

We integrated the assembly approach into the LAMS Learning Activity Management System [3] that integrates different environments for authoring, running and monitoring learning designs. The LAMS authoring environment enables authors to sequence different types of learning activities, such as discussion activities and web polls, as illustrated in Fig. 2. In the next step, learning resources can be added to the learning activities.

We have extended the LAMS authoring environment to automate, at least partially, this process. An author can create a sequence of activities or reuse an existing learning design. Suppose she wants to teach the concepts of velocity and acceleration in an inquiry based learning strategy that sequences the activities "answer questions" (a1), "vote on a list" (a2), "discuss responses" (a3), "read expert view" (a4), "discuss expert view" (a5) and "personal reflection" (a6). For activities a1 and a4 that have associated learning resources, the assembly engine generates content suggestions based on domain concepts (velocity and acceleration), instructional concepts (answer questions and read expert view) and teacher attributes (in our current prototype: language, familiar measures and weights, and typical student age range). Learning resources are retrieved on-the-fly from learning object repositories and online Web sources and shown in the content suggestions area, as illustrated in Fig. 2.

To obtain a first indication of the quality of the generated content suggestions, a small-scale experiment was conducted in April 2009 at Brigham Young University, during a post-doctoral stay of the first author of this paper. Six staff members of the Instructional Design and Technology department and six students in history and social sciences teaching were asked to reuse an inquiry based sequence and to rate the quality of the generated content suggestions. Two dimensions of quality were assessed: relevancy and accuracy. Relevancy measures whether the content suggestions are applicable and helpful for the task at hand. Accuracy is defined as the 
extent to which the content is correct, reliable and free of error. The mean for both dimensions on a 7 point scale was 6.5833 (0.51493 std dev.). Although these results are only preliminary, they indicate that participants found the generated content highly relevant and accurate.

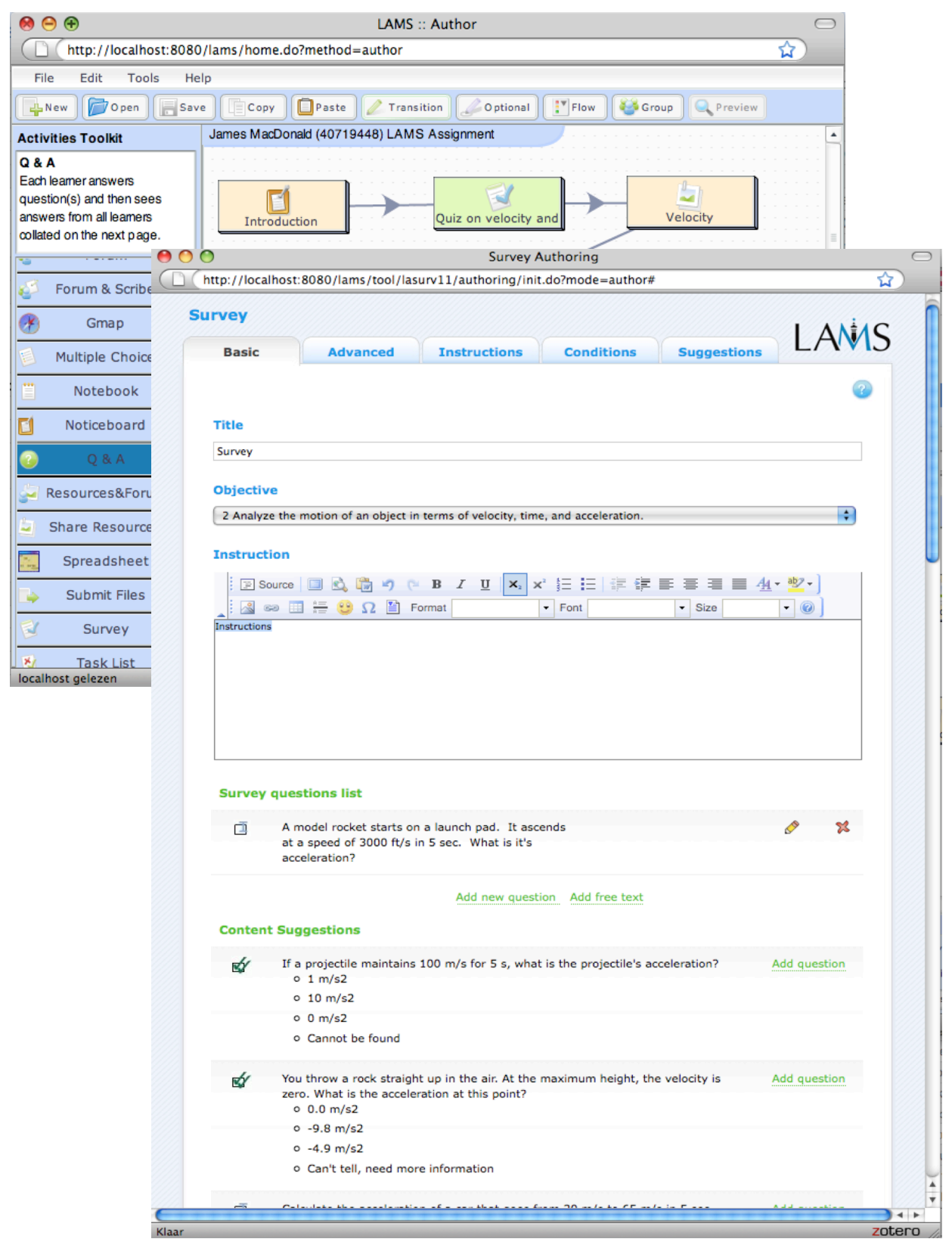

Fig. 2. LAMS plug-in 


\section{Related Work}

Reuse is considered to be an effective strategy for building high-quality learning sequences [9]. Whereas both basic and applied research have been conducted in the area of decomposing content into reusable components, little research is available on the automated reuse and assembly of content. In contrast, numerous research efforts have been made to support the development of adaptive personalized courses based on content that has been designed specifically for the course at hand [2]. Typically, multiple models are employed to support adaptivity. Dagger et al. [4] identify an instructional model, a learner model, a teacher model and a domain model. The ADAPT project [10] identifies the context of use, content domain, instructional strategy, instructional view, learner model, adaptation model and detection mechanism. The GRAPPLE project [11] identifies a domain model, a user model, a context model, an instruction model and an adaptation model. In this paper, we shifted the focus from the learner to the teacher, as automated assembly of existing learning resources requires quality control by the teacher.

Currently, there exist a range of tools to author learning sequences. Reload LD Editor [12], aLFanet LD Editor [13], CopperAuthor [14] and ASK-LDT [15] are examples of form-based editors for authoring learning designs. MOT+ [16], LD Suite [17], LAMS [3] and ACCT [4] are visual editors. Rather than developing yet another learning design environment, we incorporated our assembly strategy in the widely used LAMS authoring environment. LAMS was chosen because it provides a visual user interface that is targeted to be usable by teachers. In contrast, many of the formbased editors require good knowledge of the IMS learning design specification. In addition, LAMS was released as open source software in 2005 and has a large user community, which can potentially provide a solid basis for targeted validation.

\section{Conclusion and Future Work}

In this paper, we have presented a methodology and framework to automate the assembly of learning resources. The framework retrieves learning resources from the Web and GLOBE repositories based on a teacher model that captures teacher characteristics, an instructional model that captures the pedagogical strategy and a domain model. The approach enables the focused retrieval and aggregation of content fragments tailored to different pedagogical approaches, teacher preferences, etc. In addition, we presented a case study that integrates the approach into LAMS.

Future work will focus on validating the approach in real-world settings. One of the major motivations for integrating the approach in LAMS is the fact that LAMS is already used on a global scale. By capturing automatically the actual use by students of generated content suggestions, we will retrieve good indications of the quality of the generated learning sequences.

Acknowledgements. The research leading to these results has received funding from the European Community Seventh Framework Programme (FP7/2007-2013) under grant agreement no 231396 (ROLE) and grant agreement no 231913 (STELLAR). 


\section{References}

1. Michael D. Bush, Jonathan D. Mott (2009). The Transformation of Learning with Technology. Learner-Centricity, Content and Tool Malleability, and Network Effects. Educational Technology Magazine; March-April, 2009.

2. Vercoustre, A., and McLean, A. (2005). Reusing Educational Material for Teaching and Learning: Current Approaches and Directions. International Journal on E-Learning, 4(1):57-68.

3. Dalziel, J. R. (2003). Implementing Learning Design: The Learning Activity Management System (LAMS). G.Crisp, D.Thiele, I.Scholten, S.Barker and J.Baron (Eds), Interact, Integrate, Impact: Proceedings of the 20th Annual Conference of the Australasian Society for Computers in Learning in Tertiary Education. Adelaide, 7-10 December 2003.

4. Dagger, D., Wade, V., Conlan, O., (2005). Personalisation for All: Making Adaptive Course Composition Easy, IFETS journal of Educational Technology and Society, Special Issue on Authoring of Adaptable and Adaptive Educational Adaptive Hypermedia.

5. Maria Virvou and Maria Moundridou. Adding an Instructor Modelling Component to the Architecture of ITS Authoring Tools. International Journal of Artificial Intelligence in Education (2001), 12, 185-211

6. Wiley, David (1999). Learning objects and the new CAI: So what do I do with a learning object ? Available at http://opencontent.org/docs/instruct-arch.pdf .

7. Ternier, S., Massart, D., Campi, A., Guinea, S., Ceri, S., and Duval, E. (2008). Interoperability for Searching Learning Object Repositories: The ProLearn Query Language. D-Lib Magazine, 14(1/2).

8. Simon, B., Massart, D., van Assche, F., Ternier, S., Duval, E., Brantner, S., Olmedilla, D., and Miklos, Z. (2005). A Simple Query Interface for Interoperable Learning Repositories. In Proceedings of the 1 st Workshop on Interoperability of Web-based Educational Systems, pages 11-18.

9. Schluep, S. Modularization and structured markup for web-based learning content in an academic environment. Aachen: Shaker Verlag, 2005.

10. Garzotto, F., \& Cristea, A. I. (2004). ADAPT: Major design dimensions for educational adaptive hypermedia. In Proc. of ED-MEDIA 2004, June 21-26, 2004, pp. , 1334-1339.

11. De Bra, P., Pechenizkiy, M., van der Sluijs, K. \& Smits, D. (2008). GRAPPLE: Integrating Adaptive Learning into Learning Management Systems. In Proceedings of World Conference on Educational Multimedia, Hypermedia and Telecommunications 2008 (pp. 5183-5188). Chesapeake, VA: AACE.

12. Reload, Project website, accessed 20 April, 2009, available at http://www.reload.ac.uk/.

13. Van Rosmalen, P., and Boticario, J. (2005). Using Learning Design to Support Design and Runtime Adaptation. In R. Koper and C. Tattersall (Eds.), Learning Design. A Handbook on Modelling and Delivering Networked Education and Training. The Netherlands: Springer, 291-301

14. Van der Vegt, 2005 Van der Vegt, W. (2005). CopperAuthor. Heerlen: Open University of The Netherlands. Available from www.coppercore.org.

15. D.G. Sampson, P. Karampiperis and P. Zervas, "ASK-LDT: A Web-based learning scenarios authoring environment based on IMS Learning Design", Advanced Technology for Learning 2(4), 2005.

16. Paquette G., Lundgren-Cayrol K. , and Léonard M. (2008) The MOT+ Visual Language for Knowledge-Based Instructional Design, in Botturi and Stubs (Eds), Handbook on Virtual Instructional Design Languages.

17. Elive Learning Design, http://www.elive-ld.de/content/index_eng.html 